\title{
Childhood Obesity Declines Project: An Effort of the National Collaborative on Childhood Obesity Research to Explore Progress in Four Communities
}

\author{
Tina J. Kauh, PhD, MS,' Nicola Dawkins-Lyn, MPH, Carrie Dooyema, MSN, MPH, RN, ${ }^{3}$ \\ Carole Harris, $\mathrm{PhD}^{2}$, Jan Jernigan, $\mathrm{PhD}^{3}$, Laura Kettel Khan, $\mathrm{PhD}$, \\ Phyllis Ottley, $\mathrm{PhD}^{4}$ and Deborah Young-Hyman, $\mathrm{PhD}^{5}$
}

\section{Abstract}

Background: Recent findings show that national childhood obesity prevalence overall is improving among some age groups, but that disparities continue to persist, particularly among populations that have historically been at higher risk of obesity and overweight. Over the past several years, many jurisdictions at the city or county level across the nation have also reported declines. Little evaluation has focused on understanding the factors that influence the implementation of efforts to reduce childhood obesity rates. This article summarizes the rationale, aims, and overall design of the Childhood Obesity Declines Project (COBD), which was the first of its kind to systematically study and document the what, how, when, and where of community-based obesity strategies in four distinct communities across the nation.

Methods: COBD was initiated by the National Collaborative on Childhood Obesity Research (NCCOR), was led by a subset of NCCOR advisors and a research team at ICF, and was guided by external advisors made up of researchers, decision makers, and other key stakeholders. The research team used an adapted version of the Systematic Screening and Assessment method to review and collect retrospective implementation data in four communities.

Results: COBD found that sites implemented strategies across the many levels and environments that impact children's well being (akin to the social-ecological framework), building a Culture of Health in their communities.

Conclusions: COBD demonstrates how collaboratives of major funders with the support of other experts and key stakeholders, can help to accelerate progress in identifying and disseminating strategies that promote healthy eating and physical activity.

Keywords: childhood obesity declines; community-level; implementation; National Collaborative on Childhood Obesity Research

\section{Background}

$\mathbf{D}$ ata from the most recent National Health and $\mathrm{Nu}-$ trition Examination Survey (NHANES) indicate that nearly one in five children (18.5\%) 2 to 19 years of age in the United States had obesity in 20152016. ${ }^{1}$ Prevalence has been and continues to be even higher among those from African American and Hispanic backgrounds (e.g., $22 \%$ and $25.8 \%$, respectively, compared with
$14.1 \%$ of Whites in 2015-2016). ${ }^{1}$ The overall childhood obesity prevalence in the nation had increased significantly between 1976 and 2004. Over the last 45 years, the percentage of children and adolescents (2-19 years of age) who were considered to have obesity more than tripled, rising from $5 \%$ to over $17 \%{ }^{2}$

In response to these alarming rates, numerous strategies (i.e., policies, campaigns, programs, changes to the built environment) to increase healthy eating and physical activity

\footnotetext{
'Research-Evaluation-Learning Unit, Robert Wood Johnson Foundation, Princeton, NJ.

${ }^{2}$ Division of Health, Research, Informatics, and Technology, ICF International, Atlanta, GA.

${ }^{3}$ Division of Nutrition, Physical Activity, and Obesity, Centers for Disease Control and Prevention, Atlanta, GA.

${ }^{4}$ Division of Violence Prevention, Centers for Disease Control and Prevention, Atlanta, GA.

${ }^{5}$ Office of Behavioral and Social Sciences Research, National Institutes of Health, Bethesda, MD.
} 
have been implemented at the local, state, and federal levels to curb what has often been referred to as the childhood obesity epidemic. Potentially, as a result of these broad efforts, progress is on the horizon. Although there has been a striking increase in childhood obesity since the 1970's, NHANES data from 2003 to 2014 suggest that the rate for children overall may have stabilized at the national level. ${ }^{2,3}$ Similarly, in the past 5 years, more than 35 U.S. jurisdictions (at the local or state levels) have reported signs of progressdeclines in obesity measures among some segment of their population, including young children from low-income families, ${ }^{4,5}$ over periods ranging from 3 to 8 years. ${ }^{4-14}$

However, despite early signs of progress in curbing the childhood obesity epidemic overall, many jurisdictions have seen significant disparities in the populations that have experienced those improvements. At the local and state level, for instance, populations that typically have been at greatest risk of obesity have also been least likely to experience equal levels of progress, if any at all. ${ }^{6,7,12,14}$ While much research has informed the development or selection of strategies that jurisdictions have chosen to implement, the field's knowledge about how those strategies were implemented is limited.

Only a few prior or ongoing studies have sought to address this crucial gap. The Healthy Communities Study, for instance, has explored drivers of obesity declines using methods to measure the association between characteristics of community-level programs and policies with BMI, nutrition, and physical activity in children. ${ }^{15}$ This largescale study that includes $\sim 5000$ children in kindergarten through eighth grade and their parents provides an extensive look at the breadth of programs and policies that 130 communities nationwide have implemented and whether those implemented strategies were associated with obesityrelated outcomes.

The current study, the Childhood Obesity Declines (COBD) Project, complements the large-scale national study by using a significantly different approach with a unique goal, relying on a deeper examination in a small but diverse set of communities that experienced declines in their rates of childhood obesity to understand not only on what strategies were implemented but also how those strategies were implemented. The purpose of this article is to provide a brief overview of the National Collaborative on Childhood Obesity Research's (NCCOR) motivation for this study, the study's goals, its approach, and its contribution to the field. The other articles in this special issue present in-depth summaries and discussions of methodology, ${ }^{16}$ key findings around the settings in which healthy eating and physical activity policies and broader strategies were implemented during periods of obesity declines, ${ }^{17}$ for which specific local- and state-level policies were implemented across those different settings, ${ }^{18}$ the ways in which strategies implemented within each community reflect the social-ecological model, ${ }^{19}$ and implications for research and evaluation of community-based childhood obesity efforts. ${ }^{20}$

\section{Description of the COBD Study}

\section{Study Purpose}

While signs of progress have been growing in number across the nation in recent years, no studies have systematically assessed the quality of those reports based on the methodological strengths and limitations of sites' data collection and analysis efforts. Many of these reports have been published only in the "gray literature," such as reports released through local or state government agencies. Moreover, the methodology for collecting and analyzing obesity-related data significantly vary from one jurisdiction to another, making assessments about these reports across jurisdictions challenging. In addition, no prior studies have systematically explored the broad range of factors that facilitated (or impeded) the implementation of healthy eating or physical activity strategies in local jurisdictions or provided insight as to why progress may have been experienced by high-risk demographic groups in some communities but not in others. As such, the COBD project had three primary aims:

1. to identify jurisdictions with data on changes in obesity that met standards of quality established by the COBD project team;

2. to conduct an in-depth examination of a small number of jurisdictions that met standards of quality to identify what strategies were implemented, how those strategies were implemented, and what key stakeholders perceived to be major factors that may have facilitated or impeded progress in their jurisdiction; and

3. to understand the extent to which jurisdictions targeted their efforts toward populations at greater risk of obesity.

This study had potential to inform the field about critical implementation factors that may have influenced progress in reducing rates, particularly for children and youth at higher risk of obesity. It was also an opportunity to explore methodological adaptations, which could advance the study of community-based obesity prevention efforts. NCCOR is a collaboration of the nation's four largest funders of childhood obesity-relevant research: the CDC, the NIH, the Robert Wood Johnson Foundation (RWJF), and the USDA (see http://nccor.org for more information about the collaborative). NCCOR's mission is to accelerate progress in reducing childhood obesity. As such, the collaborative was motivated to undertake this study in an effort to advance evidence building and dissemination through a coordinated effort to address the priorities across the four NCCOR member organizations. While the study was financially supported by RWJF, NCCOR as an entity (1) provided intellectual support through the expertise of its members and its ability to convene a diverse set of experts and key stakeholders, (2) guided the development of a study that coordinated the various interests and priorities of major funders, and (3) developed opportunities to disseminate key findings that reached diverse audiences across the four member organizations. 


\section{Overview of Study}

Through funding from RWJF, ICF was contracted to conduct a qualitative study to better understand what strategies and implementation factors might have contributed to the declines reported in local jurisdictions (city or county level) in the United States, particularly among those populations at highest risk. The "COBD project team" included a research team from ICF that conducted the study as well as an NCCOR advisory group that provided guidance in the study development. The COBD project team was also complemented by an External Expert Advisory Panel, which consisted of experts in public health and research methodology, who provided input on study goals and interpretation of findings [see Kettel Khan et al. (this supplement) for a list of the external advisors].

Guided by an adapted version of the Systematic Screening and Assessment (SSA) methodology, ${ }^{21}$ the COBD project team identified 19 jurisdictions that had reported declines in childhood obesity rates at the time the study was initiated. Based on a set of exclusion and selection criteria developed by the COBD project team, ${ }^{15}$ four sites were selected: Anchorage, AK; Granville County, NC; New York, NY; Philadelphia, PA. In advance of site visits, the ICF team conducted scans of existing data sources to identify relevant strategies in school, community, childcare/early childhood education, and healthcare settings, as well as demographic characteristics of each jurisdiction. In addition, ICF distributed surveys to key stakeholders in each study community to learn more about which specific strategies were employed. Over a 1-month period in April through May of 2015 , the ICF team then conducted site visits consisting of up to 30 in-depth key informant interviews with individuals who developed, implemented, or evaluated programs and policies within those jurisdictions. More details about the methodology used to conduct the COBD project and the results of those data collection efforts are summarized in the articles in this supplement. ${ }^{15-17}$

\section{Conclusion}

The COBD project was an examination of four distinct local jurisdictions across the United States that have each experienced declines in childhood obesity rates while implementing numerous strategies to increase healthy eating and physical activity in a wide range of settings. This study was designed as a preliminary step in understanding from local key stakeholders' perspectives what factors may have influenced the impact of those strategies from conception to implementation. Its intent was not to necessarily make causal conclusions. but rather to identify potential drivers for further exploration in future research.

The findings from this study should be considered within the context of its limitations. While the study's target jurisdictions differed in terms of geography, urbanity, and populations that experienced declines, the small number of jurisdictions included in the study limits the conclusions that can be made. The study's implementation data relied primarily on qualitative methods and were collected retrospectively, in a few cases going back nearly 10 years. In addition, respondents' perspectives can bias perceptions of what factors made a difference. Furthermore, respondents' memories fade over time, potentially making them more susceptible to an individual's bias. Importantly, no causal conclusions can be made about the data.

Despite these limitations, however, the COBD project contributes to the field in several ways. Methodologically, the study demonstrates that an adapted version of the SSA method can be successfully used as a retrospective approach to assess the quality of reported COBD and employed to examine outcomes associated with community-based childhood obesity prevention efforts. Substantively, findings from the COBD project's in-depth interviews with key informants demonstrates a major tenet of the socialecological model - that the layering of strategies at multiple levels of individuals' social and ecological environments may potentially have a synergistic impact. The study also provided numerous concrete examples of factors commonly believed to positively influence implementation of prevention strategies, including the importance of stakeholder engagement, the power of a champion within the community to bring diverse sectors together and drive needed changes, and the importance of targeting efforts to those in greatest need. Finally, the COBD project highlighted how communities that demonstrated a Culture of Health - one that promotes a shared value of health for all that strives to build healthier more equitable communities, and whose members participate in cross-sector collaborations - also experienced progress in addressing a major health epidemic.

\section{Acknowledgments}

The authors thank NCCOR members, Melissa Abelev, Veronica Uzoebo, and Ruth Morgan, of the Food and Nutrition Service of USDA for their participation on the project advisory committee. They also thank the External Expert Advisory Panel for providing input in the study design. The findings and conclusions in this report are those of the authors and do not necessarily represent the official position of the CDC, NIH, USDA, RWJF, or any of the other project agencies. The Robert Wood Johnson Foundation funded this project (ID \#71772, \#73433), 50 College Road East, Princeton, NJ 08540.

\section{Author Disclosure Statement}

No author has any commercial associations that might create a conflict of interest, and no competing financial interests exist.

The authors did not report any conflicts of interest or financial disclosures. The findings and conclusions of this report are those of the authors and do not represent the official position of the Centers for Disease Control and Prevention, ICF, National Institutes of Health, or Robert Wood Johnson Foundation. 


\section{References}

1. Hales CM, Carroll MD, Fryar CD, Ogden CL. Prevalence of Obesity Among Adults and Youth: United States, 2015-2016. NCHS Data Brief, No. 288. National Center for Health Statistics: Hyattsville, MD, 2017.

2. Fryar CD, Carroll MD, Ogden CL. Prevalence of overweight and obesity among children and adolescents aged 2-19 years: United States, 1963-1965 through 2013-2014. Available at www.cdc.gov/ nchs/data/hestat/obesity_child_13_14/obesity_child_13_14.pdf (last accessed February 20, 2018).

3. Ogden CL, Carroll MD, Fryar CD, Flegal KM. Prevalence of Obesity Among Adults and Youth: United States, 2011-2014. NCHS Data Brief, No. 219. National Center for Health Statistics: Hyattsville, MD, 2015.

4. May AL, Pan L, Sherry B, et al. Vital Signs: Obesity among lowincome, preschool-aged children-United States, 2008-2011. MMWR 2013;62:629-634.

5. Pan L, Freedman DS, Sharma AJ, et al. Trends in obesity among participants aged 2-4 years in the special supplemental nutrition program for women, infants, and children-United States, 20002014. MMWR 2016;65:1256-1260.

6. Babey SH, Wolstein J, Diamant AL, et al. Patchwork of progress: Changes in Overweight and Obesity Among California 5th-, 7th-, and 9th-Graders, 2005-2010. UCLA Center for Health Policy Research and California Center for Public Health Advocacy, Los Angeles, CA, 2011.

7. Berger M, Konty K, Day S, et al. Obesity in K-8 Students-New York City, 2006-2007 to 2010-2011 School Years. MMWR 2011; 60:1673-1678.

8. Granville-Vance District Health Department. 2012 State of the County Health Report-Granville \& Vance. Available at http:// gvph.org/wp-content/uploads/2014/08/SOTCH-2012-final_12-20-12 .pdf accessed July 19, 2017.

9. New Mexico Department of Health. New Mexico Childhood Obesity Update: 2015. Available at www.tn.gov/assets/entities/ education/attachments/csh_bmi_school_summary_2012-2013.pdf (last accessed July 19, 2017).

10. Robbins JM, Mallya G, Wagner A, et al. Prevalence, disparities, and trends in obesity and severe obesity among students in the school district of Philadelphia, Pennsylvania, 2006-2013. Prev Chronic Dis 2015;12:150185.

11. The State of Alaska Department of Health and Social Services Obesity Prevention and Control Program. Alaska Obesity Prevention and Control: Student Weight Status (Anchorage Metropolitan Area). Available at http://dhss.alaska.gov/dph/Chronic/Documents/Obesity/pubs/201314WeightStatus_ASDandMSBSD.pdf (last accessed July 19, 2017).

12. Wen X, Gillman MW, Rifas-Shiman SL, et al. Decreasing prevalence of obesity among young children in Massachusetts from 2004 to 2008. Pediatrics 2012;129:823-831.

13. Zhang L, Kolbo JR, Kirkup M, et al. Childhood obesity rates in Mississippi (Prevalence and Trends in Overweight and Obesity among Mississippi Public School Students, 2005-2013). J Mississippi State Med Assoc 2014;55:80-87.

14. Robbins JM, Mallya G, Polansky M, et al. Prevalence, disparities, and trends in obesity and severe obesity among students in the Philadelphia, Pennsylvania, School District, 2006-2010. Prev Chronic Dis 2012;9:120118.

15. Arteaga SS, Loria CM, Crawford PB, et al. The Healthy Communities Study: Its rationale, aims, and approach. Am J Prev Med 2015;49:615-623.

16. Kettel Khan L, Ottley P, Harris C, et al. Childhood Obesity Declines: A different methodology. Child Obes 2018;14:S5-S11.

17. Ottley P, Dawkins-Lyn N, Harris C, et al. Childhood Obesity Declines Project: An exploratory study of strategies identified in communities reporting declines. Child Obes 2018;14:S12-S21.

18. Dooyema C, Jernigan J, Warnock A, et al. Childhood Obesity Declines Project: Review of State Level Policies. Child Obes 2018;14:S22-S31.

19. Ottley PG, Dawkins-Lyn N, Harris C, et al. Childhood Obesity Declines Project: An exploratory study of strategies identified in communities reporting declines 2018;14:S12-S21.

20. Young-Hyman D, Morris K, Kettel Khan L, et al. Childhood Obesity Declines Project: Implications for research and evaluation. Childhood Obesity 2018;14:S40-S44.

21. Leviton LC, Kettel Khan L, Dawkins N (eds). The Systematic Screening \& Assessment Method: Finding innovations worth evaluating. New Dir Eval 2010;125:7-31.

Address correspondence to:

Tina J. Kauh, PhD, MS

Research-Evaluation-Learning Unit Robert Wood Johnson Foundation 50 College Road East Princeton, NJ 08540

E-mail: tkauh@rwjf.org 